\title{
Tangence
}

\section{Les études littéraires et la fin de l'Histoire}

\section{Michel Pierssens}

Numéro 51, mai 1996

Paradigmes critiques

URI : https://id.erudit.org/iderudit/025910ar

DOI : https://doi.org/10.7202/025910ar

Aller au sommaire du numéro

Éditeur(s)

Tangence

ISSN

0226-9554 (imprimé)

1710-0305 (numérique)

Découvrir la revue

Citer cet article

Pierssens, M. (1996). Les études littéraires et la fin de l'Histoire. Tangence, (51), 157-173. https://doi.org/10.7202/025910ar d'utilisation que vous pouvez consulter en ligne.

https://apropos.erudit.org/fr/usagers/politique-dutilisation/ 


\section{Les études littéraires et la fin de l'Histoire Michel Pierssens}

Pour Marcel Muller

La critique américaine a produit, ces dernières années, un genre nouveau, que les convenances fondamentales qui règlent la discipline depuis ses débuts auraient dî interdire. Un genre où s'entremêlent, d'une façon qui ne va pas sans scandale pour beaucoup, l'analyse des textes et l'autobiograpbie, parfois assez impudique. Cependant, disent certains, la littérature - occasion de ces anamnèses - n'est-elle pas elle-même issue d'une plongée comparable et tout lecteur n'est-il pas appelé par elle à retrouver le lieu privé où repose, selon Proust, son propre "livre intérieur"? Et nombre d'universitaires de s'en autoriser pour travailler à leurs mémoires ou pour livrer les détails de leur Edipe, par où ils croient ressembler à Flaubert. Parallèlement, la critique française, si longtemps insuffisante et frivole, mais qui s'était convertie non sans arrogance dans les années soixante à la "théorie" et à la "scientificité", semble s'être faite depuis quelques années toute modestie et bumilité. La voilà qui maintenant s'ensevelit volontiers dans les tâches ingrates de l'érudition, arpentant les austères et parfois désolants chantiers de la génétique ou de l'bistoire, férue tout à coup de biograpbie et de bibliométrie, éditant à qui mieux mieux des textes de plus en plus lointains. Là où la critique américaine, entichée de cultural studies, parcourt l'archive de façon très personnelle, à la recherche de toutes les étrangetés tout en rêvant de subversion, la critique française affecte l'air absorbé des comptables balzaciens dont les minuties font un monde. Le divorce va s'accentuant de saison en saison. C'est de cette incomprébension réciproque croissante que je m'autoriserai pour introduire à mon tour dans les réflexions qui vont suivre une légère toucbe d'autobiograpbie, nécessaire pour saisir en quoi les évolutions séparées des deux traditions critiques posent problème à ceux qui, comme je le fais, ont beaucoup voyagé entre elles, tout en pensant longtemps les rapprocber. Voyages intellectuels et symboliques, mais physiques également, entre deux langues, deux systèmes universitaires, deux formes d'institution critique, mais aussi trois pays 
dont Montréal est bien, sinon la plaque tournante, du moins la curieuse interface: un monde entre deux mondes où toutes les immigrations intellectuelles cberchent leur improbable intégration. Ces réflexions ont pris forme peu à peu et ne sont en rien achevées - comme leur thème l'imposerait d'ailleurs, puisqu'il touche de manière essentielle au temps et à l'indétermination. Elles s'efforcent, de manière inévitablement décousue, de saisir ce qu'il peut $y$ avoir d'intelligible dans un parcours inauguré voilà vingt-cinq ans et qui m'a amené, comme beaucoup d'autres, à traverser des vérités successives, des pays différents, des passions périssables. Esquissées au moment de la guerre du Golfe, poursuivies pendant la guerre dans l'ex-Yougoslavie, on risquerait cependant d'y voir l'expression d'un pessimisme bistorique de bien plus vaste portée que ce que j'envisageais au point de départ. J'essaierai plus loin d'évaluer la pertinence de ces connotations millénaristes qui sont venues s'ajouter d'elles-mêmes à mon propos et de ramener les choses à des proportions moins apocalyptiques. Mais il reste que la façon dont on traite un sujet qui touche à l'bistoire ne peut pas se soustraire aux soucis du présent.

Il s'agira au fond de nous demander où nous en sommes "nous" qui sommes les gestionnaires de ce patrimoine que constitue l'bistoire des échanges intellectuels et littéraires entre l'Europe et l'Amérique. Il s'agira aussi de nous interroger sur la situation particulière où nous nous trouvons, aujourd'bui où l'eurocentrisme se trouve, aux Etats-Unis, critiqué de toutes parts et de plus en plus refusé. Plus généralement, il faudra tenter d'esquisser un bilan mais aussi signaler de nouvelles voies que pourraient emprunter les études littéraires dans leur fragile domaine. Il faudra essayer de discerner, autant que faire se peut, où nous allons, et risquer des pronostics. Valéry traitait les textes rassemblés dans Variété d'essais "quasi politiques". Avec toute la modestie qui s'impose, c'est là le modèle que j'aimerais imiter, en essayant de mêler autant que faire se peut l'anecdote et la réflexion.

$$
\text { *** }
$$

Je vais avoir l'air d'évoquer des temps préhistoriques, ou prépost-historiques, si j'essaie de rappeler de quoi l'on parlait sur les campus voilà vingt-cinq ans. En jouant au jeu de Perec, "je me souviens", la première pensée qui me vient, c'est celle-ci: “je me 
souviens du structuralisme". Vers 1970, il n'était question partout que de cela et le débat faisait rage: fallait-il ou ne fallait-il pas faire des séminaires sur cette nouvelle perversion? Directeur d'une jeune revue américaine que patronnait Barthes et qui publiait Derrida, c'est ainsi par exemple que je dus une fois infliger à un public que ces choses-là agitaient, et à un point qui nous est devenu incompréhensible, une exégèse de Lacan que j'avais réussi à condenser en deux heures - mais pas moins: l'honneur de l'avant-garde française l'exigeait. Ce même zèle missionnaire que beaucoup partagaient fit, on le sait, que la "modernité " devait l'emporter partout et très rapidement. Avec tant de succès que bien des antistructuralistes virulents de l'époque ont fini par se convertir, avec une conviction d'ailleurs si sincère que leur foi survit peut-être encore à l'écroulement de la religion ellemême.

À l'abri de l'appellation générale et impropre de "structuralisme", à côté d'un refus généralisé de l'histoire et de l'historicisme, c'était pourtant, paradoxalement, le sentiment que nous faisions l'histoire et que nous étions des "forces de progrès" qui animait l'assaut. Il y avait des bons et des méchants, des progressistes et des réactionnaires. Pour l'essentiel, c'est ce qui venait alors de France qui se trouvait du "bon" côté et venait bousculer les formalismes indigènes (le new criticism systématiquement attaqué et dévalué), les subjectivismes, les relativismes et autres humanismes décadents et bourgeois. Chacun le sait, cette histoire-là est bien finie - à la fois parce que la page est tournée (sans cependant que le chapitre suivant paraisse avoir vraiment commencé) et parce que nous ne pouvons plus croire à ce genre d'histoire.

Nous avions à l'époque toutes les réponses - nous n'avions en fait que des réponses. Nous sommes aujourd'hui confrontés en revanche à de multiples questions que l'on s'étonne de retrouver aussi insistantes alors qu'elles paraissaient il y a peu encore faire partie de l'archive historique d'un mode de pensée désormais caduc - c'est-à-dire "classées" une fois pour toutes. Il redevient urgent, par exemple, de réfléchir à ce que nous pouvons penser encore avec des notions comme celles-ci : progrès et décadence; commencement et fin; rupture et conservation... 
160

Mais je vais laisser cette interrogation provisoirement en suspens, parce qu'il me faut d'abord essayer de situer un peu plus précisément la nature de l'écart que je vois grandir chaque jour sur ces sujets-là entre les deux mondes, l'Europe et les Etats-Unis, entre lesquels nous avions l'habitude de voyager sans visa. Des univers qui ont fonctionné pendant un certain temps de façon synchrone mais dont le décalage devient de plus en plus sensible.

Du côté français, le malaise est évident, le désarroi intellectuel général et parfaitement perceptible. Michel Serres en avait donné, dans son discours de réception à l'Académie française, un exemple assez frappant :

Quelle philosopbie de l'histoire me permettra d'embrasser, dans la clairvoyance et la pitié, ce siècle barbare et fondateur dont nous ne savons si les progrès foudroyants balancèrent les plus archaïques régressions? ${ }^{1}$

Le problème tient, au moins partiellement, à l'état de la France, du français, des Français, à l'apparition diffuse d'un nouveau "mal du siècle". Ce "mal" français (dans un sens différent de celui qu'impliquait ce titre d'Alain Peyrefitte) se nourrit d'un renouveau de notions comme celle de décadence, que l'on voit revenir dans les éditoriaux comme principe d'explication hâtif, dans les livres comme objet d'étude. Il s'y ajoute un sentiment très fort de pourrissement de l'Université, le tout sur fond d'effacement, voire d'effondrement des croyances politico-théoriques (structuralisme, sémiotique, psychanalyse, etc.). On s'étonne comme d'un archaïsme, voire d'une faute de goût, de voir le livre de Pierre Macherey, Á quoi pense la littérature? paraître dans une collection dont le titre est "pratiques théoriques" - une expression désormais aussi datée que le marxisme-léninisme ou le mur de Berlin.

Pour ne nous en tenir qu'aux domaines qui sont les nôtres, la vérité est qu'il existe en France aujourd'hui un authentique déficit d'écoles et d'adversaires. Pas de catéchisme en formation, pas d'ennemi à abattre, pas de place forte à prendre - le contraste avec la situation américaine est saisissant. La France vit depuis plusieurs années une sorte de Restauration molle dont Mitterand

1 Le Monde, 3-4 février 1991, p. 11. 
aurait été le Charles X (même l'opposition est déprimée). Chacun trouve de plus en plus plus difficile de poser une affirmation quelconque à propos de quoi que ce soit. Parmi les symptômes les plus évidents du mal je ne citerai que l'aplatissement des héros intellectuels des années 1960 chez Sollers et Kristeva Femmes ou Les samouraïs ne sont guère plus qu'un assez mauvais feuilleton qui n'a émoustillé qu'un temps les midinettes vieillissantes du Quartier Latin. Où sont d'ailleurs les œuvres littéraires qu'on pourrait citer à l'appui d'une analyse du monde contemporain? Je jalouse Kermode qui, dans The Sense of an Ending, pouvait encore faire sans complexe le va-et-vient entre histoire et littérature en commentant à la fois l'Apocalypse, La nausée ou l'emploi du temps, et trouver dans des romans des ressources fondamentales pour une synthèse philosophique et anthropologique de grande dimension.

On est frappé de voir aux Etats-Unis, tout au contraire, l'intensité des combats théoriques. De fait, l'Europe s'éloigne - à moins que ce ne soit l'Amérique, question de perspective - et il faudrait bien sûr analyser, d'un point de vue européen, ce qui apparaît comme un triomphe de l'illusion idéologique (quelque chose comme une nouvelle version du livre de Zinoviev: vers l'avenir radieux par la déconstruction). Je ne me lancerai pas dans une telle analyse. Je me contenterai de rappeler comment réagissent les Français en voyant avec un mélange d'amusement et d'incrédulité se rejouer aux Etats-Unis aujourd'hui les débats européens d'il y a vingt ans, scandés par les mêmes slogans et appuyés sur les mêmes noms - Derrida, Lacan, Deleuze, Barthes, les divers féminismes - et ceci en un temps où les Français n'en finissent pas d'élaborer des bilans comme celui, d'ailleurs magistral, que la revue Le Débat avait proposé en 1988 sous le titre "Notre histoire. Matériaux pour servir à l'histoire intellectuelle de la France, 1953-1987" et sur lequel je reviendrai.

Comment apprécier le sens de cette reprise américaine des vieux scénarios français? S'agit-il d'une répétition un peu dérisoire ou au contraire de l'épanouissement trop longtemps attendu d'une révolution culturelle avortée ailleurs ${ }^{2}$ ? Cet apparent décalage intellectuel entre la scène française et la scène américaine se

2 Autre symptôme: l'élimination du programme doctoral de recherches féministes de Paris-8 dirigé depuis plus de vingt ans par Hélène Cixous. 
162

trouve symbolisé très nettement, me semble-t-il, par la dissymétrie des débats: la critique du new historicism ici, au nom tantôt du modernisme et tantôt du post-modernisme, l'épanouissement sans résistance de la critique génétique là-bas. La conséquence en est, pour rester toujours dans notre champ, que le travail empirique se fait désormais en Europe, le délire idéologique aux ÉtatsUnis - ce qui constitue une nouveauté! L'Europe croit à nouveau à l'Histoire et au Sujet et cette croyance n'a plus d'ennemis - il n'y a donc pas de combat; l'Amérique, en revanche, y croit de moins en moins et se bat dêsormais contre elle-même. Il faut en convenir : il y a de quoi rester perplexe. Personne ne veut être en retard d'une régression, évidemment - mais qui le serait en l'occurrence: la France en revenant au "canon", à l'histoire, à la biographie ou les États-Unis en revenant en-deça des "acquis" de l'histoire littéraire positive?

Mais poser la question en ces termes a-t-il encore un sens? Peut-être la plus grande conquête du post-modernisme est-elle en fait que personne ne peut plus être en retard sur personne: tous les passés ont également de l'avenir. Pour qu'il y ait retard, il faut que le monde, l'histoire ou la société soient articulés en zones bien distinctes entre lesquelles se déploient des circulations parfois fluides et parfois plus visqueuses, qui donnent elles-mêmes un rythme différencié à la temporalité, au sens du temps consubstantiel à l'identité que chaque zone se construit par rapport à ses voisines. En d'autres termes (ceux de l'historien Krzysztof Pomian), pour qu'il y ait du retard ou de l'avance, il faut qu'il y ait des "tribus" - or, elles n'existent plus. Il n'y a plus d'idéologues français ni de maîtres-penseurs, c'est donc entendu - mais y en a-t-il pour autant aux États-Unis? La diffusion des thèmes importés a-t-elle entraîné la constitution de sectes et de chefs? Rien n'est moins sûr. Pourquoi par exemple la déconstruction n'est-elle pas devenue le déconstructionnisme organisé ou le derridisme militant? Le grand mystère: pourquoi n'y a-t-il pas un Journal of Deconstruction ou une School of Deconstruction, privée et très chère? Est-ce dérobade des chefs ou déficit de foi? Toujours est-il qu'ici aussi le tribalisme s'efface, malgré les apparences. Après l'ère européenne des chefs de clan, voici l'heure des dieux cachés, ou des imams disparus (comme chez les Chiites): Derrida n'est-il pas invisible bien qu'omniprésent (il y a toujours un nouveau texte de Derrida sur tout)? Nous sommes bel et bien passés d'une organisation tribale 
(celle qui avait triomphé dans le mouvement structuraliste) à un système "viral" (c'est l'explication que donnerait sans doute Baudrillard de la déconstruction). Le mystère de la percolation des idées dans le réseau hyper-complexe du système universitaire rejoint celui de l'épidémiologie du sida, dont je n'ai pas besoin de souligner à quel point il constitue la grande matrice métaphorique de notre temps.

Il y a de nombreux avantages à cet effacement des modes antérieurs d'organisation du champ intellectuel, mais aussi des inconvénients. Il en résulte entre autres, comme le dit Krzysztof Pomian, une réduction de la capacité de nuire des paranoilaques et des mégalomanes - "D'où, dans la culture, la fin des prophètes, ce qui est bien, mais aussi, ce qui est triste, l'extrême rareté de grandes ambitions théoriques dans les sciences humaines et sociales, de philosophies vertigineuses, d'œuvres littéraires et artistiques exaltantes par leur radicalité." ${ }^{3}$ Sommes-nous, pour le dire autrement, gagnés définitivement par le repentir - où Baudrillard voit le trait majeur du post-moderne?

$$
* *
$$

Voilà des perspectives bien sombres, dira-t-on, et qui sentent furieusement l'abandon aux vertiges fin-de-siècle. De fait, il semble que nous en soyons arrivés au point (dans une perspective à la française) où seul nous resterait, selon la formule connue, "l'ennui interminable de l'avenir". Ce serait vrai tout au moins si nous devions en rester à la mélancolie, et nous enliser dans le travail de deuil inachevè à l'égard des marvelous seventies - notion empruntée encore une fois à Baudrillard. Mais malgré les bilans, les rétrospectives, les autopsies qui se multiplient, il est clair que l'Europe est mieux équipée pour penser le commencement que pour analyser la fin. Cela provient, selon Hannah Arendt, de la force des traditions révolutionnaires, car "les révolutions, dit-elle, sont les seuls événements politiques qui nous placent directement, inéluctablement, devant le problème du commencement " 4 . Aussi est-ce maintenant le moment de revenir à ce que j'avais laissé en suspens tout à l'heure, pour tenter de mieux

3 Le Débat, n ${ }^{\circ}$ 60, mai-août 1990.

4 Hannah Arendt, Essai sur la rétolution, Paris, Gallimard, 1963. 
164

cerner ce sentiment que quelque chose s'achève, et peut-être découvtir quelque moyen d'y échapper.

Et d'abord, la fin des grands problèmes et des "grands récits", pour parler comme Lyotard quand il veut caractériser la postmodernité, doit-elle s'interpréter comme l'ouverture de notre horizon sur une authentique fin de l'bistoire, ou bien comme ce que Baudrillard appelle "l'hystérésis du millenium", c'est-à-dire le retour du syndrome fin-de-siècle typiquement européen? Je renvoie quant à ce syndrome, largement étudié récemment, aux excellents travaux d'Hillel Schwart ${ }^{5}$, à son article intitulé "Finde-siècle Fantasies: a brief history of the end of Time" paru dans The New Republic ${ }^{6}$ ou encore à la somme de Marc Angenot: 1889. Un état du discours social ${ }^{7}$. Il faut en revanche se poser la question suivante: mais de quoi parle-t-on quand on parle de "fin de l'histoire", et qu'est-ce que cela peut bien signifier pour nous, dans notre champ? Et y a-t-il un lien essentiel entre l'état de la littérature et l'êtat du monde, l'état de la connaissance et l'état de notre discipline?

Si la fin de l'histoire signifie, comme le veut Fukuyama ${ }^{8}$, qu'il n'y ait plus d'idéologie qui vaille et que tous acceptent désormais la transcendance indiscutable du marché et de la démocratie consommatrice, que nous reste-t-il alors à faire: commémorer le passé sans y toucher, ou bien l'étudier d'une autre façon, encore mal discernable, mais en espérant malgré tout de cet exercice un gain cognitif, et dans ce cas: lequel? L'étude de la littérature n'aura ni le même sens ni le même avenir si l'on considère que l'essentiel a été fait ou si l'on considère au contraire qu'il reste à accomplir des progrès fondamentaux dans l'organisation des sociétés et de leur relation au passé, en particulier leur passé cul-

5 Hillel Schwartz, Century's End: a cultural history of the fin-de-siècle; from the 990 s to the 1990s, New York, Doubleday, 1990.

630 juillet-6 août 1990.

7 Marc Angenot, 1889. Un état du discours social, Longueuil, Québec, Le Préambule, 1990. Cf. également Daniel Milo: "La fin du siècle n'aura pas lieu ", Le Débat, op. cit., et André-Clément Decouflé, qui analyse six techniques pour prédire l'avenir: divination, prophétie, utopie, science-fiction, futurologie, prospective, dans L'An 2000. Une anti-bistoire de la fin du monde, Paris, Gallimard-Julliard, coll. "Archives", 1975. À quoi l'on ajoutera le collectif Rbétoriques fin de siècle, Paris, Christian Bourgois, 1992.

8 Francis Fukuyama, "Entering Post-History", New Perspectives Quaterly, VI, 3, automne 1989. 
turel, dont nous sommes bon gré mal gré les fonctionnaires et les gestionnaires.

Avant de tenter d'esquisser une issue, il me faut cependant évoquer au moins un aspect de ce que pourrait signifier pour nous l'avènement d'un monde qui ressemblerait à celui que prédit Fukuyama. Un en sens, on peut dire que ce monde est déjà là. Notre fin de l'histoire à nous, n'est-ce pas en effet ce qui est en train de se mettre en place à une vitesse vertigineuse, parallèlement à la mondialisation de l'économie: le marché total du texte, l'archivage absolu, la mémoire instantanée de toute la littérature et de tous les temps sous forme électronique dans des projets comme la Text Encoding Initiative ou le National Center en cours de constitution'? Il n'y aurait aucun sens à parler de fin de l'histoire et de perspectives pour l'avenir des études littéraires si l'on n'évoquait pas la dimension technologique de la question. Le sujet est désormais incontournable. On se rappellera que l'humanisme heideggerien s'était fondé dans une large mesure sur une critique de la technique; or, l'humanisme d'aujourd'hui est inconcevable sans la technologie, aussi faut-il s'interroger sur ce que nous allons en faire ainsi que de ses progrès - des progrès qui vont être radicaux. Comme le dit sans hésiter le vice-président américain Gore: "electronics are to our age what coal and iron were to the Industrial revolution".

Peut-être sommes-nous déjà entrés aujourd'hui dans un univers qui ressemblerait très fort à celui que décrit Paul Virilio, complètement bouleversé par l'instrumentalisation de la vitesse de la lumière: il y désormais simultanéité radicale entre tous les événements - nous vivons dans l'instantané, ce qui compliquera formidablement le problème des transferts de savoir et de pouvoir entre générations académiques. Mais une question plus grave se pose: que restera-t-il à transférer? Du hardware ou du software? des données ou des idées? du patrimoine ou des procédures? des livres ou une rhétorique? L'informatisation ne fera-t-elle pas que les ouvres seront de plus en plus remplacées par leur représentation par des morceaux choisis sélectionnés par des procédures de recherche très éloignées des méthodes de lecture que nous connaissons depuis le Moyen-Âge? L'œuvre, devenue

9 Cf. Michel Pierssens et Luciano Floridi, "Le désir de tout savoir", Qu'est-ce qu'on ne sait pas?, Paris, Gallimard, Découvertes, Rencontres philosophiques de l'Unesco, 1995, p. 92-93. 
166

archive, demeurera strictement virtuelle: plus jamais elle ne sera lue au complet - et ses limites auront d'ailleurs depuis longtemps perdu leur netteté, grâce à la saisie de tous les états, tous les manuscrits, tous les brouillons. Nous disposerons d'une mémoire répartie, totale, mais sans "sujet" constituant. La critique sera remplacée par la liste bibliographique, à la rigueur par l'abstract. Les spécialistes seront devenus avant tout des techniciens des études littéraires qui sauront manipuler des concepts, des classes, des étiquettes, des traces et des marqueurs et en interpréteront les relations et les agencements. Ce sera pour de bon la fin de la critique comme reconstruction (et même de la déconstruction - puisque celle-ci suppose encore un texte, bien que déjà largement virtuel, depuis toujours déjà artefactuel).

Si ce scénario a quelque chance de se réaliser - ce que je crois - ne va-t-il pas devenir nécessaire de penser des formes encore mal appréhendables d'écriture, d'expression, d'argumentation ${ }^{10}$ ? Quelle forme aura la communication savante en littérature? Celle du "poster" comme chez les scientifiques? Celle de la note archivable, sans tissu conjonctif pour la relier à une démarche singulière? C'est dès maintenant que se met en place le diptyque qui oppose les données de fait à l'éditorial - ce qui ne fait que généraliser à nos domaines le triomphe des formes de communication médiatiques, puisqu'il s'agit au fond de la même structure que celle qui juxtapose dans les média l'objet graphique complexe et l'objet verbal simplifié : le modèle et le commentaire, la figure et la légende. Déjà plus personne ne lit les articles imprimés (sans même parler des livres) s'ils dépassent un certain nombre de pages. Bien des éléments des publications d'aujourd'hui font déjà figure de restes fossiles d'une évolution antérieure, dont la fonction est oubliée, comme les aigrettes ou les palmes d'animaux formés lors d'une autre ère géologique. Comment en tirer la conséquence? S'il paraît difficile sur le plan pratique de suivre la suggestion de Baudrillard qui propose tout simplement de faire l'économie de la fin du siècle et.de passer directement au suivant, on pourrait au moins - pour hâter la réalisation des nouvelles formes de la recherche - soutenir le projet, repris par George Steiner ${ }^{11}$, d'une sorte de moratoire sur les publications universi-

10 Cf. Michael Heim, Electric language: a pbilosophical study of word processing, New Haven, Conn. Yale University Press, 1987.

11 George Steiner, Présences réelles, Paris, Gallimard, coll. "Les Essais", 1991. 
taires - quelque chose comme un véritable désarmement critique multilatéral, pendant que se mettent en place les nouvelles structures électroniques.

L'incapacité où se trouve la critique de se faire lire, en proportion directe de sa capacité à se faire imprimer, relève sans doute de causes extrinsèques, en partie socio-techniques comme je viens de le suggérer. Mais cet échec de la critique ${ }^{12}$ tient peutêtre aussi à des causes intrinsèques: peut-être s'agit-il d'abord d'un échec stylistique. La critique universitaire ne sait pas encore s'écrire comme on écrit aujourd'hui : par formules fortes, en s'appuyant sur des systèmes de métaphores technologiques (ou biotechnologiques) tombées dans le domaine public et faisant désormais partie des savoirs ambiants, de ce que tout le monde sait reconnaître comme porteur de sens. Peut-être est-ce là la force de Baudrillard, car il réalise ce que Deleuze et Guattari n'avaient fait qu'esquisser, en mobilisant la figure du rhizome ou celle du corps sans organes. Inversement, c'est la faiblesse de Derrida qui, depuis la différance avec un $a$, n'a pas inventé une seule bonne formule. L'opposition type, dans le domaine français, peut être symbolisée par ce qui distingue le style du Monde et le style Libé, Voir et Devoir, comme le trahit la consonance-dissonance de leurs titres (je suis prêt à parier qu'on y verra, rétrospectivement, une ligne de fracture majeure dans la langue et l'expression, l'un des grands moments du siècle - incommensurablement plus important que les réformes de l'orthographe aussitôt enlisées, débordées par le mouvement autonome, combien plus puissant, de la langue de tous, que personne ne saurait contrôler). C'est aussi bien toute la différence entre le TLS et le VLS: dans un cas, l'information est là, mais intransmissible, du fait de son emballage; dans le second, elle est là aussi, mais avec de la communication en plus - le medium, plus le message - en attendant une édition de Wired consacrée à la littérature.

J'en conclus que l'un des premiers moyens d'échapper à l'obsolescence qui guette tous nos discours, qui risquent de s'enfoncer d'un coup dans l'archaïsme comme cela s'est produit si souvent déjà dans l'histoire, ce serait d'abord d'apprendre à écrire pour notre temps. Vaste tâche, que je ne peux aborder ici.

12 La notion d'échec n'a pas encore été recyclée, alors qu'elle avait beaucoup servi à certaines époques antérieures. Surveillons son retour: il signifiera un tournant. 
Puisque nous voilà confrontés à des perspectives qui peuvent paraître apocalyptiques, il importe de retrouver notre équilibre, et le moment est sans doute venu d'esquisser quelques propositions, aussi minces soient-elles. L'effondrement des avant-gardes a ceci de positif qu'il nous laisse le champ libre pour tout essayer: pour la première fois depuis longtemps, nous sommes à nouveau libres de nos choix.

Aujourd'hui se font jour, en dépit de tout, de nouvelles interrogations sur l'objet littérature, sur le temps qui le transforme, sur les discours qui le lient à son temps — tout cela grâce à l'émancipation que nous vivons depuis une quinzaine d'années, même si nous la percevons d'abord sous sa forme dépressive: fin du marxisme, fin du freudisme, fin du sémiologisme, même si l'on voit ici ou là des tentatives pour reconstruire des discours monologiques, féminismes radicaux ou néo-marxismes divers (menace de la "ligne juste", du "politically correct "). Plutôt que la déplorer, il faut en fait chercher à approfondir cette émancipation par rapport à tous les -ismes, comme d'ailleurs par rapport aux formes religieuses de la déconstruction. Le mot peut surprendre, mais j'insiste là-dessus, avec le même étonnement que Marcel Gauchet:

Il y aura eu entre-temps pour compliquer le tableau la greffe réussie de la "déconstruction" sur les études littéraires américaines. C'est au pays de l'individu-roi que le décentrement structural et la dissolution discursive trouveront un épanouissement dont la conversion au "souci de soi", concédé in extremis par Foucault, les a privés sur leur sol natal. Malin qui nous expliquera comment la francité quintessentielle de cet hybride aura pu devenir un moment l'idiome messianique des campus. ${ }^{13}$

La dérive possible vers les intégrismes théoriques tient à beaucoup de causes, difficiles à sonder, mais l'une d'elles - la plus profonde - ne serait-elle pas la fin d'un pouvoir propre de la littérature, définitivement marginalisée, et plus encore peut-être des spécialistes de cette littérature? Sans vouloir opposer au messianisme de la théorie une autre forme de "mission" tout à fait désuète, ne reste-t-il pas que les littéraires sont ou devraient être porteurs, non seulement d'une tradition à transmettre mais plus 
encore d'un type de questionnement à communiquer, par nature radicalement réfractaire à tous les intégrismes? Je ne crois pas, en effet, que beaucoup de choix nous soient encore ouverts. Il faut opposer la critique, solidement appuyée sur les problématiques historiques d'aujourd'hui, développée dans un style nouveau, à la "théorie", l'ouverture au repli, la lecture des textes à la lecture des ravaudages de seconde main (comment ne pas être d'accord avec Steiner là-dessus?); retrouver les écrivains - même si la littérature et les littérateurs semblent avoir perdu leurs ambitions, au moins chez les Français - mais rappelons-nous ce que Michel Raimond nous a dit de La mort du roman, toujours annoncée depuis la fin du $\mathrm{XIX}^{\mathrm{e}}$ siècle et toujours attendue, et ce que nous savons de la poésie, toujours donnée pour morte et toujours renaissante. Baudelaire disait déjà, comme toute la seconde moitié de son siècle: "le monde va finir" - mais c'est précisément là qu'il a trouvé la ressource d'une poésie nouvelle. Il faudrait donc nous ressaisir: aller vers la nouvelle littérature, c'est-à-dire à la fois les textes les plus anciens et les textes les plus nouveaux, les plus risqués; ne pas jeter tous les outils ni renoncer à la recherche critique interdisciplinaire. Parier par exemple sur l'extension des savoirs littéraires en retrouvant le sens et le rôle critique de la littérature dans la constitution des savoirs, des représentations, des institutions, bref : de la culture en général - tout ce que je résume sous l'appellation d' "épistémocritique "14.

Plus concrètement, la question est, encore et toujours: que devons-nous enseigner? Dans quelle voie engager les chercheurs pour que leur recherche soit pertinente et leur assure un avenir intellectuellement productif? Tout ce que le "modèle" français peut offrir aujourd'hui, c'est la critique génétique alliée à la sociocritique, bien floues l'une comme l'autre dans leurs fondements théoriques comme dans leurs conclusions. Cela produit des résultats mais n'est guère exaltant. On peut en outre difficilement imaginer, du point de vue américain, une démarche qui soit plus liée au "canon". Qui va se donner en effet la peine de suer sur les manuscrits d'un(e) inconnu(e) ou d'un marginal? Quel éditeur voudra en financer la publication d'une édition critique? Et ne voilà-t-il pas longtemps déjà qu'on n'enseigne plus (aux ÉtatsUnis toujours) tout ce qu'on nous avait pourtant enseigné

14 Michel Pierssens, Savoirs à l'œuvre. Essais d'épistémocritique, Presses universitaires de Lille, 1990. 
170

naguère si rigoureusement: ni l'histoire de la langue, ni les principes de la philologie, ni la différence entre manuscrit et texte édité, ni l'histoire littéraire, ni l'histoire tout court?

Devant ces difficultés et ces incertitudes, je ne vois pas d'autre voie, pour l'avenir immédiat, que celle de la tentation de l'histoire. Jouer avec cette tentation, c'est en un sens se demander de quel côté sont aujourd'hui les Lumières et se demander si l'enseignement littéraire peut avoir à nouveau quelque chose à voir avec leur projet. Mais il faut alors faire de l'bistoire sans bistoricisme, comme on peut essayer de faire de la religion sans croyance (ce que propose Michel Deguy en parlant de "croyance en suspens", pour y puiser un nouveau sujet: le sujet d'Annonciation), si du moins l'on définit l'historicisme comme la croyance au progrès dans l'histoire, au sens où Popper le définit "the doctrine that it is the task of the social sciences to propound historical prophecies, and that historical prophecies are needed if we wish to conduct politics in a rational way" ${ }^{15}$. C'est bel et bien d'une histoire nouvelle qu'il s'agit, comme le souligne Marcel Gauchet:

Mais c'est sous le signe d'une anthropologie attachée à faire saillir l'étrangeté du passé et de mentalités établissant l'universelle historicité de l'humanité vivante, enfance, amour et mort, que la discipline [l'Histoire] a significativement d'abord percé les profondeurs multiples du devenir se substituant à l'assurance linéaire du développement. C'est d'autre chose qu'il s'agit dorénavant, quand il faut éclairer la provenance de ces dimensions politiques, juridiques, intellectuelles qui font notre identité originale de modernes. Cela demande d'évoluer dans des durées qui bousculent le cloisonnement académique des époques et des spécialités. Cela exige de nouer des paramètres dont l'intrication transgresse le mieux établi de nos partages, celui qui scinde la matérialité des faits de l'idéalité des représentations.

Un mouvement est parti qui n'est pas près de s'interrompre. La figure de l'histoire sur laquelle nous nous reposions depuis qu'il y a pensée de l'histoire s'est dérobée sous nous. Bon gré, mal gré, en conscience ou sans le savoir, nous ne couperons pas à la tâche d'en reconstruire une. ${ }^{16}$

15 Karl Raimund Popper, "Prediction and prophecy in the social sciences", Conjectures and refutations: the growtb of scientific knowledge, New York, Harper \& Row, coll, "Harper Torchbooks", 1965, p. 336. 
Il y a là, dans notre champ, la possibilité d'ouvrir un chantier nouveau, marqué par la nécessité de reprendre le travail empirique, dans l'interaction concrète des disciplines, loin des rêveries des années soixante sur une multidisciplinarité purement métaphysique. Comme le dit Clifford Geertz à propos du and dans l'expression "anthropology and history": "Take care of the conjunctions and the nouns will take care of themselves. " ${ }^{17}$ Les études littéraires, quant à elles, s'étaient fondées au $\mathrm{xx}^{\mathrm{e}}$ siècle sur l'hypothèse qu'il pouvait y avoir une continuité intelligible dans la succession des formes et des projets littéraires dans le temps ce qui n'allait pas de soi ${ }^{18}$. Ces mêmes études littéraires se sont ensuite préoccupées des phénomènes de rupture (la fin, le renouveau; les anciens et les modernes; les avant-gardes et la subversion, etc.) Mais ne serait-il pas temps qu'elles se soucient à nouveau un peu plus désormais de la liaison et du passage? Ne serait-il pas temps par exemple de recycler la notion de transition, puisque nous paraissons être dans un de ces moments ambigus où chacun sent que quelque chose se termine mais aussi que ce qui va prendre la suite n'a pas encore de figure? Le $\mathrm{XVIII}^{\mathrm{e}}$ siècle avait inventé le mot, mais c'est le $\mathrm{xtX}^{\mathrm{e}}$ siècle qui a fait longtemps ses délices de cette notion, avant de sombrer dans le sentiment crépusculaire fin-de-siècle, en attendant la fin du monde. La Transition, comme le rappelle Frank Kermode d'après l'Apocalypse, c'est ce temps hors du temps, comme suspendu, où le Temps ne coule plus mais s'étale, ce moment problématique qui vient juste avant la fin et juste après que l'histoire se soit arrêtée. Sans doute est-ce pour cela que Baudelaire s'est intéressé à ce qu'il nomme "les dévergondages propres aux époques de transition ", dans le Salon de 1846, rejoint par Gobineau qui fait dire à l'un des personnages des Pléiades (ce n'est qu'un exemple de ce qui se dit à l'époque):

17 Clifford Geertz, "Anthropology and History", New Literary History, XXI-2, 1990.

18 C'est ce dont témoigne par exemple ce passage d'un essai classique d'Ozanam sur Dante: "Les littératures se succèdent : il s'agit de savoir si elles se lient et se continuent; si, à côté de ces instincts poétiques qui partout s'éveillent d'eux-mêmes, il y a une discipline savante qui constitue l'art que les peuples se transmettent, toujours enseignés, toujours enseignants, n'accomplissant qu'une même ouvre, une même destinée. Et, pour poser la question en des termes plus courts, il s'agit de savoir s'il existe une tradition dans les lettres." Dante et la philosopbie catbolique au xul ${ }^{e}$ siècle, $7^{e}$ éd., 1895. 
172

Monsieur, je tombe d'accord avec vous que beaucoup de choses de ce temps sont odieuses, mais leur ouvre destructive est nécessaire pour préparer la place nette aux perfections qui viendront ensuite. En d'autres termes, et pour me servir d'une phrase courante, nous sommes dans une période de transition. 19

La morale que j'en tire est qu'il n'est peut-être pas mauvais d'aller chercher appui dans cette période même pour trouver de quoi combattre un tel sentiment. Rappelons-nous ce qu'en disait par exemple Renan dans L'avenir de la science: "Il est superficiel d'envisager l'histoire comme composée de périodes de stabilité et de périodes de transition. C'est la transition qui est l'état habituel." C'est lui encore qui écrit:

De même, loin que le monde moral ait reçu un coup mortel de la destruction des vieilles chimères, la méthode la plus réaliste est celle qui nous mènera aux plus éblouissantes merveilles, et jusqu'à ce que nous ayons découvert d'ineffables splendeurs, d'enivrantes vérités, de délicieuses et consolantes croyances, nous pouvons être assurés que nous ne sommes pas dans le vrai, que nous traversons une de ces époques fatales de transition, où l'humanité cesse de croire à de chimériques beautés pour arriver à découvrir les merveilles de la réalité. Il ne faut jamais s'effrayer de la marche de la science, puisqu'il est sûr qu'elle ne mènera qu'à découvrir d'incomparables beautés.

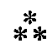

En conclusion, et pour dire les choses d'une façon moins lyrique, si le post-modernisme signifie l'extension de l'empire de la fin, alors j'opte pour un pari sur l'avenir du modernisme, puisqu'il est avant tout mouvement vers l'inconnu dans toutes les directions. Michel Serres appelait dans Passage du Nord-Ouest à la constitution d'un "tiers-instruit"; il me paraît indispensable de lui adjoindre maintenant ce qui fera de lui, tout aussi bien, un tiersétant, un voyageur d'un nouveau genre cherchant un chemin non plus seulement dans l'espace hypothétique de l'entre-deuxmondes, l'ancien et le nouveau, mais à travers le temps étrange qui sépare désormais le continent Europe du continent Amérique. $\mathrm{Vu}$ depuis cette transition essentielle, épistémique autant qu'onto- 
logique, "tout homme appartient à deux ères" comme le disait Valéry, qui ajoutait:

D’un côté, un passé qui n'est pas aboli ni oublié, mais un passé duquel nous ne pouvons à peu près rien tirer qui nous oriente dans le présent et nous donne à imaginer le futur. De l'autre, un avenir sans la moindre figure. Nous sommes, chaque jour, à la merci d'une invention, d'un accident, matériel ou intellectuel. ${ }^{20}$ 